\title{
Contents
}

The Deutsches Elektronen-Synchrotron

by J.R. Schneider, P. Söding,

G.-A. Voss, A. Wagner, B.H. Wiik

DESY - Zeuthen

91

Status of the HERA Facility

Physics at HERA

Neutrino Astrophysics

Fixed-Target Experiments at HERA

HASYLAB

Research and Development for Linear Colliders

Co-operation Essential

Anticipating Researchers Europe

92

93

94

96

99

100

104

105

106

NUCLEAR PHYSICS: Advancing on Several Fronts 110

FACILITIES: EUPRO Urges Access for First-timers

111

EPS Executive Meeting

112

CONDENSED MATTER: Call for Nominations

112

- Europhysics Notes

113

LHC Vote; VIRGO agreement;

National societies; Research networking

\section{European Physical Society}

Europhysics News is the journal of the European Physical Society that acts through Divisions, Sections and Groups to promote collaboration among physicists. Subscription price: SFR 135-- per annum.

\section{Editorial Board}

P. Bochsler, Bern University

S. Ciliberto, ENS, Lyon

A.-J. Dianoux, ILL, Grenoble

Editor: P.G. Boswell

Exec. Sec.: G. Thomas

T. Jarlborg, Geneva University

J. Schacher, Bern University

M. Siegrist, EPFL, Lausanne

President: N. Kroó KFKI, Budapest

Editorial and Advertising

EPS Secretariat, P.O.Box 69

$\mathrm{CH}-1213$ Petit-Lancy 2, Geneva

Telephone: +41(22) 7931130

Telefax: $\quad+41(22) 7931317$

E-mail: epnews@cernvm.cern.ch

Advertising is charged according to space, on a sliding scale ranging from SFR 2700.- for a full page to SFR 700.- for a one-sixth page. Rates for four-colour and cover advertising, and series insertions, on application. Special rates for Associate Members of EPS; $25 \%$ reduction for announcements of situations vacant.
Deadline for advertising orders:

first week of month of publication.

Subscriptions \& EPS Fees:

Société de Banque Suisse,

Geneva; Acc. No. 164.899

Swiss Post Office Acc.: CCP

Geneva 12-19107-4

German Post Office Acc.: Postbank

Karlsruhe 1801-30-754

Eurocheque to EPS, Geneva

EPS Budapest Secretariat

Nádor u. 7, H-1051 Budapest

Telephone: $\quad+36(1) 1173510$

Telefax: +36 (1) 1176817

\section{Cover illustration}

The many faces of DESY. Clockwise from the upper left -

The NT-200 pilot neutrino detector experiment: a pair of detector modules are being lowered into Lake Baikal.

Preparing a nine-cell niobium radio-frequency cavity: the development of high-gradient superconducting cavities is carried out in the framework of the TESLA collaboration.

The H1 Collaboration: members of HERA's H1 Collaboration in front of the open detector.

HASYLAB: a wide-angle view of the experimental hall showing the instrumentation at several synchrotron radiation beam-lines.

The HERMES gas-target experiment: a view inside the target cell.

programme will probably start in December so it is the time to start thinking about applications. Conscious that one should not expect Brussels to fund everything, we set a small ball rolling this month with an overview of human capital and mobility. The various EU programmes now have established clientele for whom there are generally few surprises between successive Frameworks. Nonetheless, details change so it is often useful to review programmes so that potential applicants avoid wasting time.

Scientists will be able to apply to several specific programmes for project support (and maybe to all the programmes if one's interests extend far enough). The European Commission has not yet taken a broad-brush look at where specific disciplines such as physics are involved so it is difficult to give priorities. All one can do is swing through the jungle, pointing out the major features and giving guide-lines on how to pin-point tasty fruit.

An area close to many physicists' hearts seems set for major changes. It is high performance computing and networks (HPCN) which came under the Directorate-General for industry (DG-XIII) when ESPRIT was transfered from DG-XII (science) in 1993. Buried deep within one of ESPRIT's successors in the 4th Framework is a targeted activity to promote applications of high-performance computers and of distributed systems. It will be organized as a "focused cluster" - an approach pioneered in ESPRIT that unites just about everything (projects, joint enterprises, networks, supplier and user associations, national initiatives, the EUREKA programme, international co-operation, dissemination, training...).
A look at the only operating focused cluster (the Open Microprocessor Systems Initiative, OMI) and ESPRIT's present work in HPCN (see ESPRIT Report 1993/4) gives an idea of what the HPCN cluster may try to achieve. It could, for instance, promote an object-orientated neural network programming language or a high-performance operating system as a world-wide standard (OMI is promoting, for example, the Eurocell Library, as a highspeed interconnection between computer systems, and the low-power ARM microprocessor used by Apple).

All this seems a long way from what most scientists see as a vital aspect of HPCN, namely low-cost, high-performance communications. Perhaps the research community has had it easy as there was a ready ear in Brussels while interests often literally ran in parallel (in parallel computing). Things have moved on (the EU will soon have nine ZEUS centres equipped with state-of-the art parallel computers to develop applications). Scientists are now seen as "calling for a pan-European network without having the resources to pay for it".

The Report of the High Performance Networking Requirements Group issued by DG-XIII in April highlights this unfortunate gap. The group, in defining industrial requirements, recommended stimulating the uptake of distributed computing applications which use advanced networks. It claims there is a viscous circle for users other than the "leading edge research community" involving "little articulated demand - so little provision - so little articulated demand."

The group made its recommendations while being fully aware that: no companies are licensed to provide trans-national links in Europe; that Europe's international leased lines are 10-times more expensive than transcontinental leased lines in the US; that none of the Post Office monopolies abide by an EC Directive that leased-line charges should be based on actual costs; that the US as a result of the so-called Gore bills will have a nation-wide broadband National Research and Education network of say $600 \mathrm{Mbit} / \mathrm{s}$ capacity in place by $1995 / 6$ (Europe has a few national/regional lines moving to $34 \mathrm{Mbit} / \mathrm{s}$ ); that the explosive growth of Internet will continue as it converts to predominantly commercial use.

The recommendations are naturally presented as "candidates for scope outside national programmes" since "our recommendations should complement national initiatives". Or as Riccardo Perissich, the DirectorGeneral of DG-XIII, writing in the ESPRIT 1993/4 report puts it: "The new technologies ... being developed (in ESPRIT) ... are areas for which effort at a European level is appropriate...; so as to provide critical mass for effective R\&D; to ensure proper co-ordination ...; and to create the best conditions for technology transfer." If there is a chicken and egg problem in a vital area, these approaches seem a poor way to solve it.

P.G. Boswell 